\title{
Ab Initio Simulation of the Absorption Spectra of Photoexcited Carriers in $\mathrm{TiO}_{2}$ Nanoparticles
}

\author{
Francesca Nunzi, ${ }^{a}{ }^{b}$ Filippo De Angelis ${ }^{a, c}$ Annabella Selloni ${ }^{*}$ \\ ${ }^{a}$ Computational Laboratory of Hybrid/Organic Photovoltaics (CLHYO), CNR-ISTM, via Elce di \\ Sotto 8, I-06123 Perugia, Italy. \\ ${ }^{\mathrm{b}}$ Department of Chemistry, Biology and Biotechnologies, via Elce di Sotto 8, I-06123 Perugia, \\ Italy. \\ ${ }^{\mathrm{c}}$ CompuNet, Istituto Italiano di Tecnologia, Via Morego 30, 16163 Genova. \\ ${ }^{\mathrm{d}}$ Department of Chemistry, Princeton University, Princeton, NJ-08544, USA. \\ * Corresponding author. E-mail: francesca.nunzi@unipg.it; aselloni@princeton.edu
}

\section{Supporting Information}

\section{S1. Computational Methods}

S2. Analysis of the absorption spectra of photoexcited electrons and holes 


\section{S1. Computational Methods}

The $\left(\mathrm{TiO}_{2}\right)_{38}$ cluster used to model $\mathrm{TiO}_{2}$ NPs was generated by appropriately 'cutting' an anatase slab exposing the majority (101) surface. ${ }^{1}$ Following the work by Persson et al., ${ }^{2}$ we consider a neutral stoichiometric $\mathrm{TiO}_{2}$ cluster with no saturating atoms or groups at the edges, ${ }^{3,4}$ which well reproduces the electronic and structural properties of $\mathrm{TiO}_{2} \mathrm{NPs} .{ }^{5}$ The $\left(\mathrm{TiO}_{2}\right)_{38}$ cluster is constituted by two layers with alternating rows of 5- and 6-fold coordinated titanium atoms, and 4fold Ti sites at the edges. Geometry optimizations were performed on the neutral $\left(\mathrm{TiO}_{2}\right)_{38}$ cluster in a closed shell electronic state to determine the equilibrium geometry of the ground state $\left(\mathbf{S}_{\mathbf{0}}\right)$, and on the charged anion $\left(\mathrm{TiO}_{2}\right)_{38}{ }^{-}$and cation $\left(\mathrm{TiO}_{2}\right)_{38}{ }^{+}$clusters in an open shell doublet spin configuration, to obtain the equilibrium geometries of the NP with a single excess electron and a single excess hole $\left(\mathbf{D}_{\mathbf{1 , e}}\right.$ and $\left.\mathbf{D}_{\mathbf{1 , h}}\right)$.

The hybrid B3LYP functional together with an all electron $6-31 \mathrm{G}^{*}$ basis set was employed, and spin polarization was included for systems with unpaired electrons. Water solvation effect were included by means of the Conductor-like Polarizable Continuum Model (C-PCM). ${ }^{6}$ We also carried some additional calculations at the B3LYP/3-21G* level of theory. All calculations were carried out using the Gaussian 09 program package. ${ }^{7}$ The transient absorption spectra of $\mathrm{e}^{-}$and $\mathrm{h}^{+}$in the $\left(\mathrm{TiO}_{2}\right)_{38} \mathrm{NP}$ were simulated at the TDDFT level (B3LYP/631G* and B3LYP/3-21G*) by computing the lowest $100 \mathrm{D}_{1}-\mathrm{D}_{1}$ excitations of the charged anion $\left(\mathrm{TiO}_{2}\right)_{38}{ }^{-}$and cation $\left(\mathrm{TiO}_{2}\right)_{38}{ }^{+}$ clusters in their relaxed $\mathbf{D}_{1, \mathbf{e}}$ and $\mathbf{D}_{1, \mathbf{h}}$ geometries. To evaluate the effect of the relaxation on the absorption spectra, we also computed the vertical electronic excitations of $\left(\mathrm{TiO}_{2}\right)_{38}{ }^{-}$and $\left(\mathrm{TiO}_{2}\right)_{38}{ }^{+}$ by employing the equilibrium $\mathbf{S}_{\mathbf{0}}$ geometry of the neutral $\left(\mathrm{TiO}_{2}\right)_{38} \mathrm{NP}$. In the calculation of the optical spectra, the 100 lowest spin-allowed $\mathrm{D}_{1}-\mathrm{D}_{1}$ transitions were taken into account, up to an excitation energy of $\sim 2$ and $3 \mathrm{eV}$ for electron and hole, respectively. As a reference, we also computed the ground state absorption spectrum of the neutral $\left(\mathrm{TiO}_{2}\right)_{38} \mathrm{NP}$ and compared with the experimental absorption spectrum of Reference ${ }^{8}$, see Figure S1. The onset is computed at $323 \mathrm{~nm}$ 
and the oscillator strength increases with decreasing wavelength, in good agreement with the experimental outcome. Transition energies and oscillator strengths have been interpolated by a Gaussian convolution with a width $\sigma=0.2 \mathrm{eV}$. The computed excitations have been analyzed in terms of contributions from the individual molecular orbitals (MOs). Due to the unrestricted formalism employed in the TDDFT, the overall percentage contributions of the configurations to the resulting excited state TDDFT wavefunction sometimes exceeded $100 \%$, without affecting the composition of the excited state. 

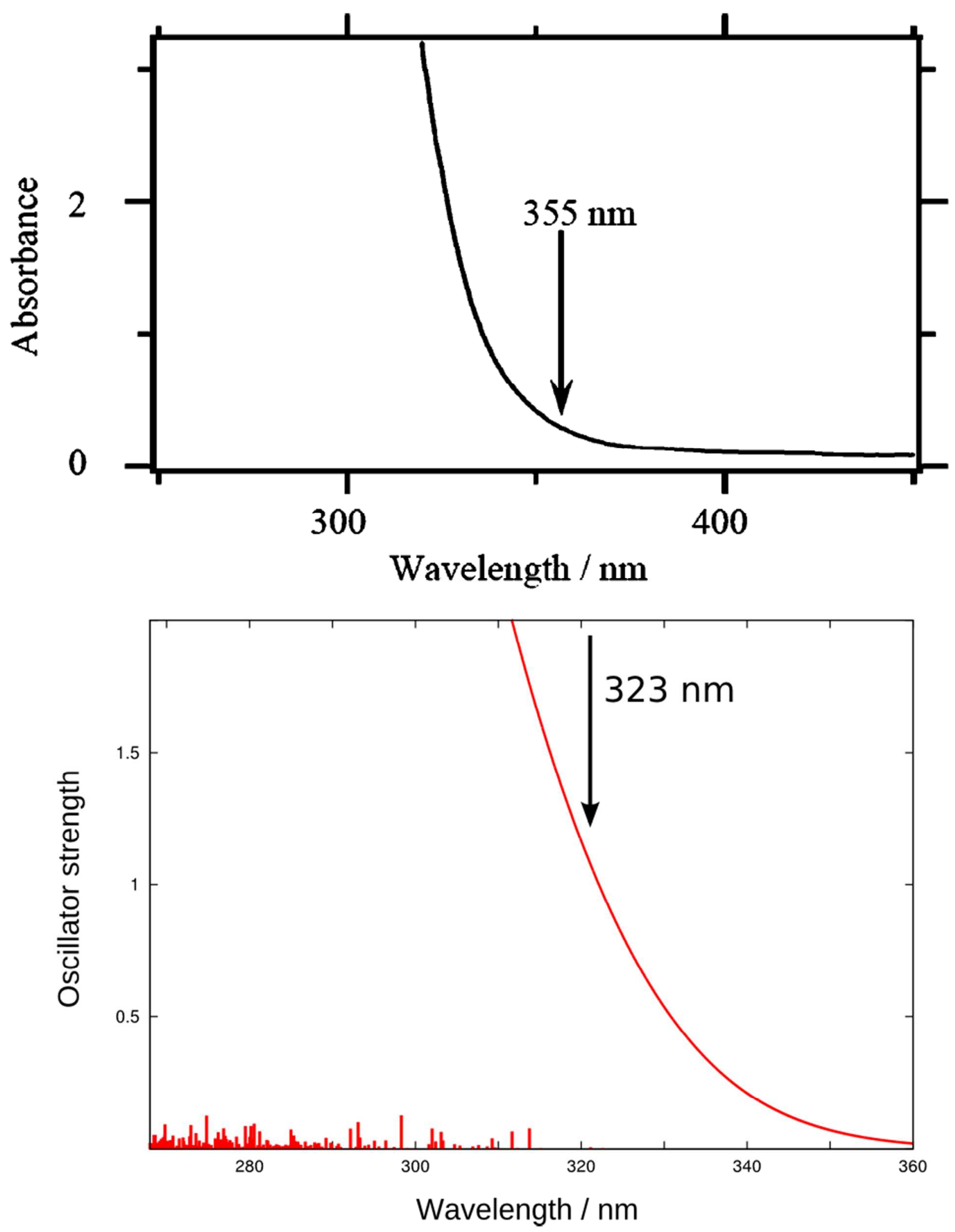

Figure S1. Top: experimental ground state absorption spectrum of $\mathrm{TiO}_{2}$ nanocrystalline film reproduced in part from $\operatorname{Ref}^{8}$ with permission of The Royal Society of Chemistry. Bottom: ground state absorption spectrum of the $\left(\mathrm{TiO}_{2}\right)_{38}$ neutral NP computed at the $\mathrm{B} 3 \mathrm{LYP} / 3-21 \mathrm{G}^{*}$ level of theory in water solvent. The Gaussian broadening in the computed spectrum is $\sigma=0.20 \mathrm{eV}$. 


\section{S2. Analysis of the absorption spectra of photoexcited electrons and holes}

To investigate the effect of the basis set on the absorption spectra of photoexcited charge carriers, we performed TD-B3LYP calculations of the lowest 100 excitations of $\left(\mathrm{TiO}_{2}\right)_{38}{ }^{-} /\left(\mathrm{TiO}_{2}\right)_{38}{ }^{+}$using both the $3-21 G^{*}$ and $6-31 G^{*}$ basis sets with the geometries optimized at the B3LYP/3-21G* and B3LYP/6-31G* levels of theory. As shown in Figures S2-S3, both basis sets reproduce the essential features of the experimental TAS spectra of Furube and co-workers. ${ }^{7,9,10,25}$ In particular, the TDDFT spectra computed with the $3-21 \mathrm{G}^{*}$ and $6-31 \mathrm{G}^{*}$ basis sets on the same B3LYP/6-31G* optimized geometry show only minor differences, while the TD-B3LYP/3-21G* spectra on the B3LYP/3-21G* geometry show smaller intensities and extend over a greater wavelength range, notably for the trapped holes, confirming that the shape of the absorption depends significantly on the cluster geometry. Nonetheless, the smaller $3-21 G^{*}$ basis set reproduces well the essential features of the absorption spectra computed with the larger 6-31G* basis set. Accordingly, in the following we report a detailed analysis of the absorption spectra of trapped charge carriers computed at the TD- B3LYP/3-21G* level of theory. 

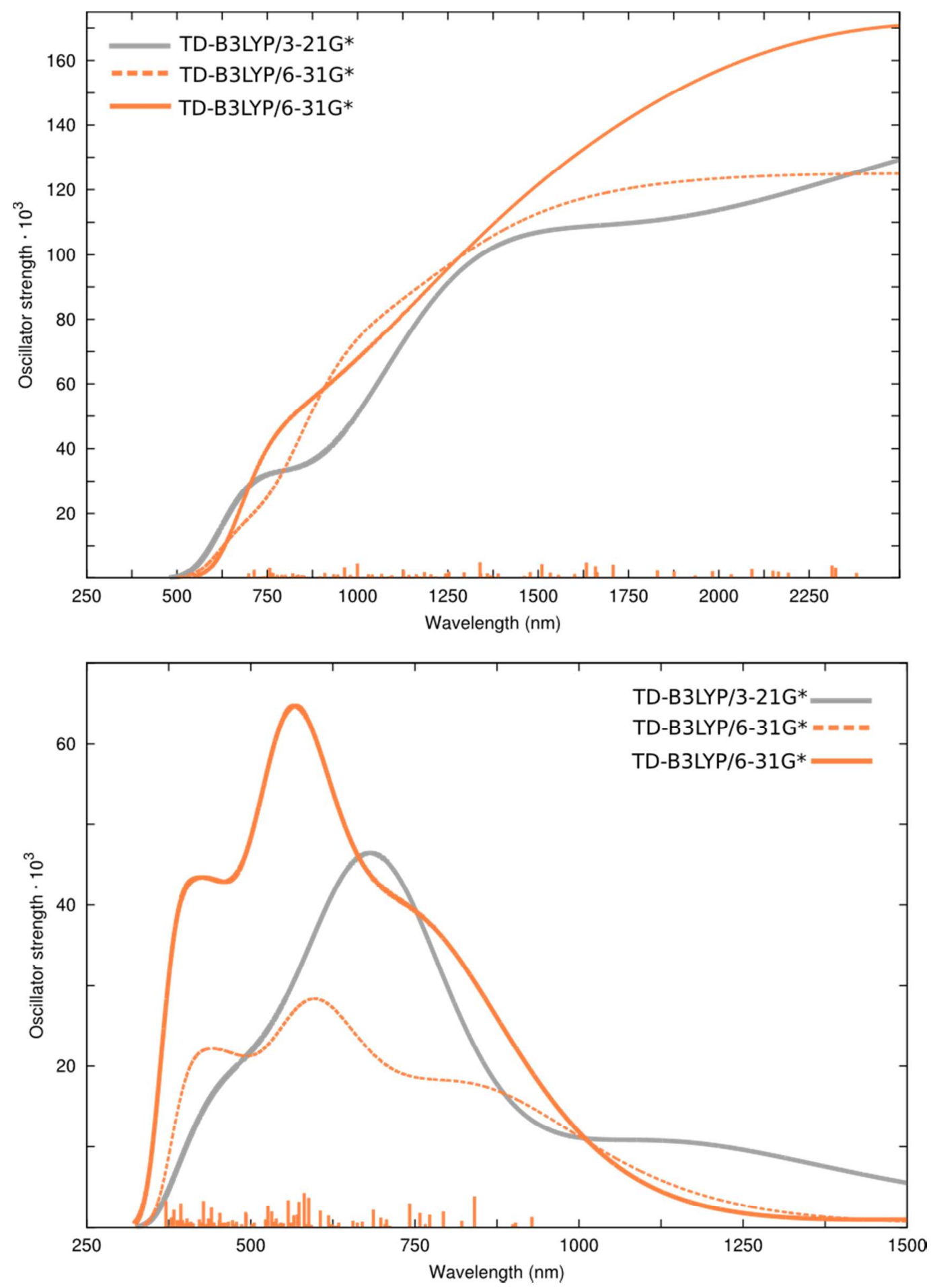

Figure S2. Absorption spectrum of the $\left(\mathrm{TiO}_{2}\right)_{38}{ }^{-}$cluster used to describe a photoexcited electron, calculated in the unrelaxed $\mathbf{S}_{\mathbf{0}}$ (top) and relaxed $\mathbf{D}_{\mathbf{1}, \mathrm{e}}$ (bottom) geometries. All calculations were performed at the TD-B3LYP level of theory in water solvent using $3-21 \mathrm{G}^{*}$ and $6-31 \mathrm{G}^{*}$ basis sets. The dashed line refers to the geometry optimized at the B3LYP/3-21G* level of theory. 

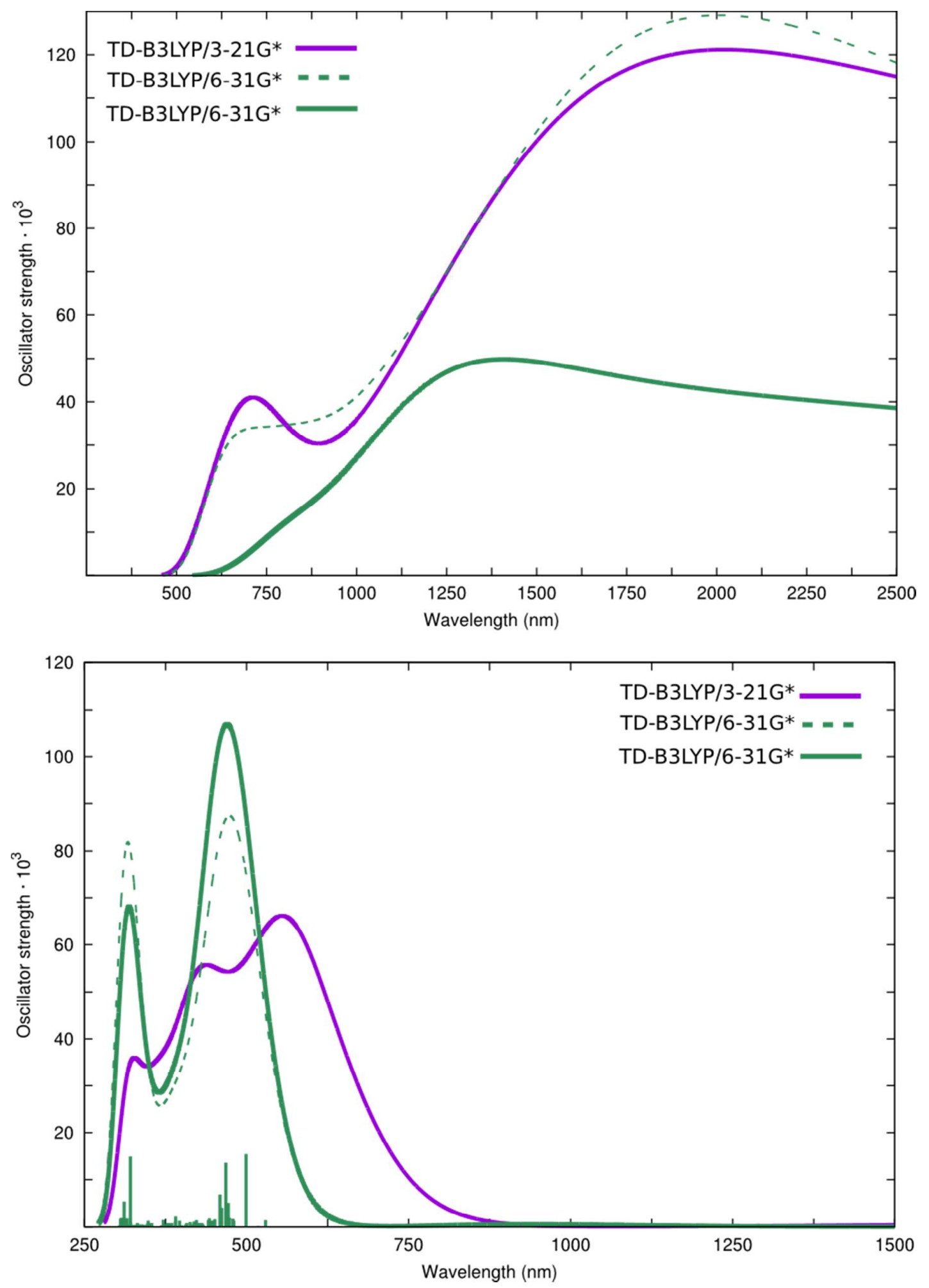

Figure S3. Absorption spectrum of the $\left(\mathrm{TiO}_{2}\right)_{38}{ }^{+}$cluster used to describe a photoexcited hole, calculated in the unrelaxed $\mathbf{S}_{\mathbf{0}}$ (top) and relaxed $\mathbf{D}_{\mathbf{1}, \mathbf{h}}$ (bottom) geometries. All calculations at the TD-B3LYP level of theory in water solvent with $3-21 \mathrm{G}^{*}$ and $6-31 \mathrm{G}^{*}$ basis sets. The dashed line refers to the geometry optimized at the B $3 \mathrm{LYP} / 3-21 \mathrm{G} *$ level of theory. 


\section{S2.1. Absorption spectra of photoexcited electrons}

Energies and oscillator strengths (f) for selected excitations of $\left(\mathrm{TiO}_{2}\right)_{38}$ in the relaxed $\mathbf{D}_{\mathbf{1}, \mathbf{e}}$ geometry, computed at the TD-B3LYP/3-21G* level of theory, are reported in Table S1 and Figure S4, together with the composition in terms of the most relevant transitions and the involved MOs. Analysis of the absorption spectrum of a trapped $\mathrm{e}^{-}$shows that the near IR part $(800-2500 \mathrm{~nm})$ is constituted by only 7 excitations ( 3 with $\mathrm{f} \cdot 10^{-3}>0.5$ ), while the remaning computed 93 excitations all fall in the visible part $\left(800-350 \mathrm{~nm}, 32\right.$ excitations with $\left.\mathrm{f} \cdot 10^{-3}>0.5\right)$, with energy differences between adjacent excitations smaller than $0.1 \mathrm{eV}$. Overall, the absorption spectrum of the trapped electron shows a very broad low intensity $\left(f \cdot 10^{-3}=11\right)$ band centered at $1100 \mathrm{~nm}$ and a high intensity peak ( $\left.\mathrm{f} \cdot 10^{-3}=46\right)$ centered at $680 \mathrm{~nm}$. Analysis of the composition of the computed excitations shows that for all transitions the initial state is the $\operatorname{SOMO}(723 \alpha)$, while the final states are among the unoccupied MOs above LUMO, formally constituting the CB. The excitations in the near IR, constituting the low intensity band, show an unexpected composition in terms of the final states of the electronic transitions, since MOs lying from $0.3 \mathrm{eV}$ up to $1 \mathrm{eV}$ are involved, despite the low energy of the transitions. The highest peak of the trapped $\mathrm{e}^{-}$absorption spectrum is mainly composed by electronic transitions (8-18) from the SOMO to unoccupied MOs (LUMOLUMO+10) lying within $0.3 \mathrm{eV}$ from the bottom of the $\mathrm{CB}$, with partially localized electron density. With increasing excitation energy, the contribution to the electronic transitions of higher unoccupied MOs increases. In particular, we verified that excitations in the range $670-480 \mathrm{~nm}$ mostly involve as final states MOs ranging from LUMO+12 $(-2.37 \mathrm{eV})$ to LUMO+50 $(-1.57 \mathrm{eV})$. The computed spectrum also shows a shoulder at $460 \mathrm{~nm}$ with $\mathrm{f} \cdot 10^{-3}=18$, originated by excitations from the SOMO to unoccupied MOs lying between $1.1-2.0 \mathrm{eV}$ above the LUMO, see, e.g., excitation $\mathrm{D}_{75}\left(445 \mathrm{~nm}, \mathrm{f} \cdot 10^{-3}=1.3\right)$. 
Table S1. Excitation energy $(\mathrm{E}, \mathrm{eV})$, wavelength $(\lambda, \mathrm{nm})$, oscillator strength $\left(\mathrm{f} \cdot 10^{-3}\right)$ and composition of some representative doublet excitations $\left(\mathrm{D}_{\mathrm{n}}\right)$ in terms of $\alpha \mathrm{MOs}(\mathrm{SO}=\mathrm{SOMO} ; \mathrm{L}=$ LUMO) for the $\left(\mathrm{TiO}_{2}\right)_{38}{ }^{-}$cluster in the relaxed $\mathbf{D}_{1, \mathrm{e}}$ geometry, computed at the B3LYP/3-21G* level of theory in water solvent.

\begin{tabular}{|c|c|c|c|c|}
\hline$D_{n}$ & $E(e V)$ & $\lambda(\mathrm{nm})$ & $f \cdot 10^{-3}$ & Composition \\
\hline 3 & 1.09 & 1137 & 3.2 & $\begin{array}{l}65 \%(\mathrm{SO} \rightarrow \mathrm{L}+13)+25 \%(\mathrm{SO} \rightarrow \mathrm{L}+15)+19 \%(\mathrm{SO} \rightarrow \mathrm{L}+8)+12 \% \\
(\mathrm{SO} \rightarrow \mathrm{L}+14)\end{array}$ \\
\hline 10 & 1.62 & 764 & 3.6 & $\begin{array}{l}44 \%(\mathrm{SO} \rightarrow \mathrm{L}+4)+24 \%(\mathrm{SO} \rightarrow \mathrm{L}+3)+16 \%(\mathrm{SO} \rightarrow \mathrm{L}+5)+16 \%(\mathrm{SO} \\
\rightarrow \mathrm{L}+6)+13 \%(\mathrm{SO} \rightarrow \mathrm{L}+26)\end{array}$ \\
\hline 15 & 1.74 & 712 & 5.2 & $\begin{array}{l}21 \%(\mathrm{SO} \rightarrow \mathrm{L}+9)+14 \%(\mathrm{SO} \rightarrow \mathrm{L}+34)+13 \%(\mathrm{SO} \rightarrow \mathrm{L}+11)+11 \%( \\
\mathrm{SO} \rightarrow \mathrm{L}+21)\end{array}$ \\
\hline 17 & 1.78 & 696 & 3.3 & $98 \%(\mathrm{SO} \rightarrow \mathrm{L}+7)+14 \%(\mathrm{SO} \rightarrow \mathrm{L}+16)$ \\
\hline 24 & 1.92 & 644 & 2.1 & $\begin{array}{l}23 \%(\mathrm{SO} \rightarrow \mathrm{L}+13)+23 \%(\mathrm{SO} \rightarrow \mathrm{L}+18)+22 \%(\mathrm{SO} \rightarrow \mathrm{L}+45)+15 \% \\
(\mathrm{SO} \rightarrow \mathrm{L}+14)\end{array}$ \\
\hline 39 & 2.16 & 574 & 1.6 & $28 \%(\mathrm{SO} \rightarrow \mathrm{L}+31)+27 \%(\mathrm{SO} \rightarrow \mathrm{L}+36)+14 \%(\mathrm{SO} \rightarrow \mathrm{L}+27)$ \\
\hline 75 & 2.79 & 445 & 1.3 & $\begin{array}{l}38 \%(S O \rightarrow L+66)+28 \%(S O \rightarrow L+67)+26 \%(S O \rightarrow L+68)+19 \% \\
(S O \rightarrow L+81)\end{array}$ \\
\hline
\end{tabular}



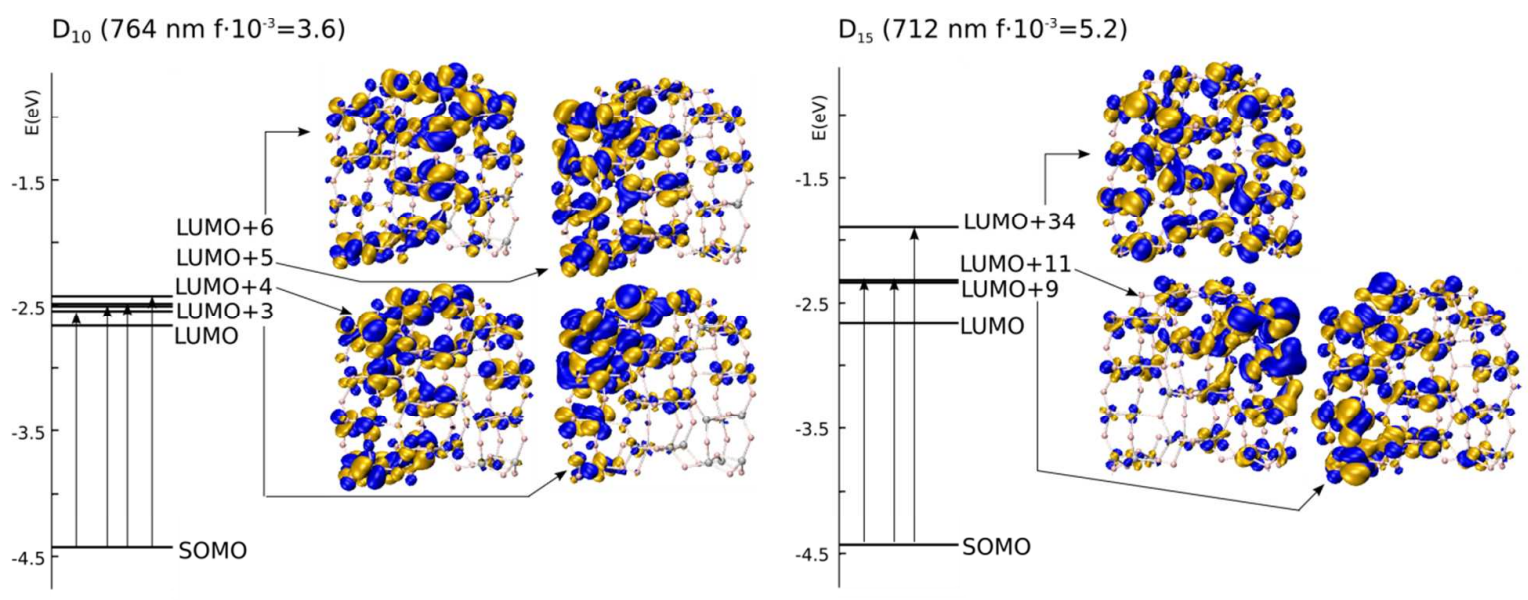

$D_{17}\left(696 \mathrm{~nm} \mathrm{f} \cdot 10^{-3}=3.3\right)$
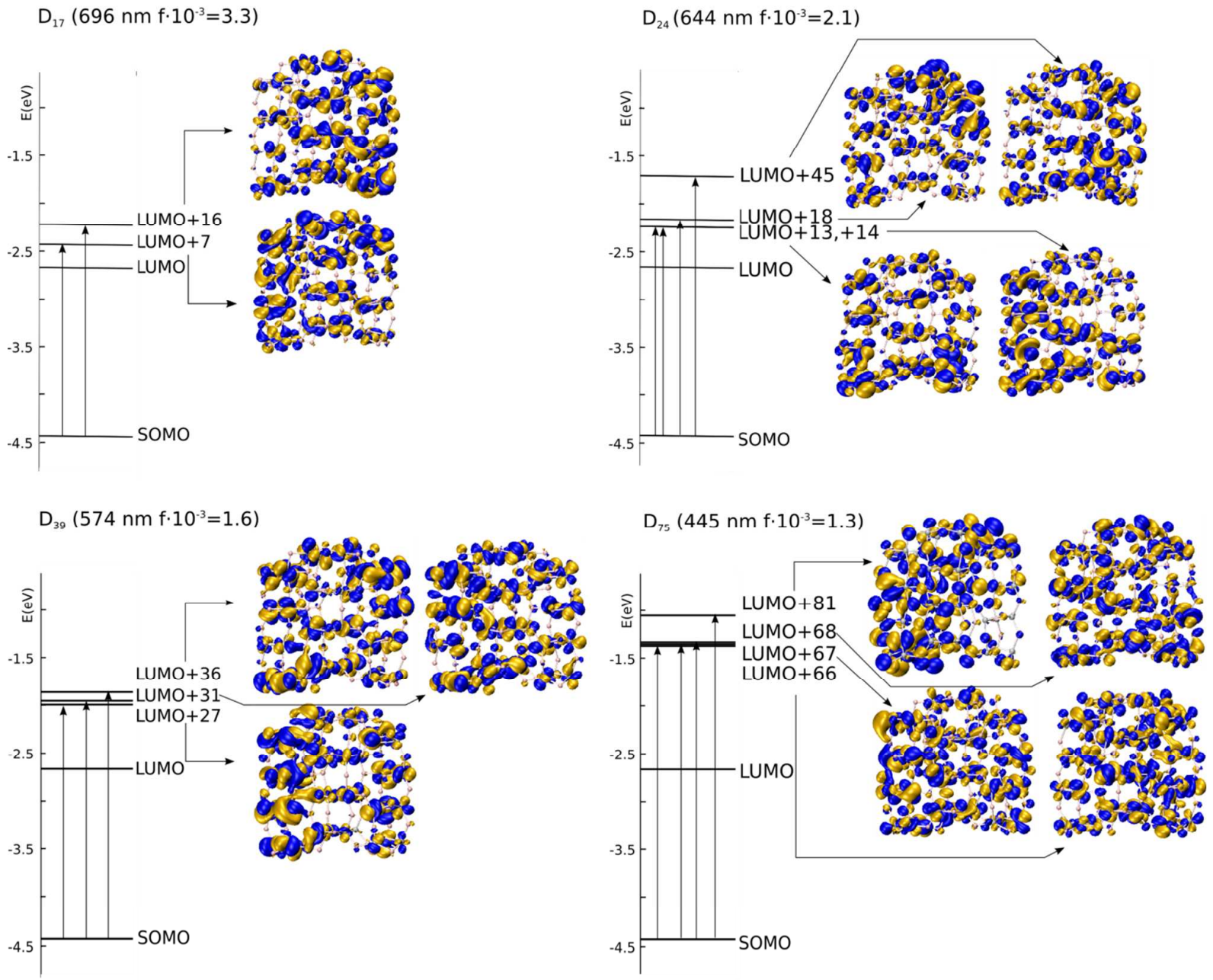

Figure S4. Representative excitations contributing to the absorption spectrum of a trapped electron in the relaxed $\mathbf{D}_{1, \mathrm{e}}$ geometry in terms of electronic transitions from the SOMO to unoccupied LUMOs, computed at the TD-B3LYP/3-21G* level of theory. The electron wavefunctions of the involved LUMOs are also shown (isovalue $\left.=0.01 \mathrm{e} / \mathrm{au}^{3}\right)$. 
As shown in Figure S2, the computed absorption spectrum of electron in the unrelaxed $\mathbf{S}_{\mathbf{0}}$ geometry is substantially different from that in the relaxed $\mathbf{D}_{1, \mathrm{e}}$ geometry, being constituted by a large numer of excitations in the IR (ca. 60) and a few (ca. 20) in the visible, up to $650 \mathrm{~nm}$. Overall, the absorption spectrum in the unrelaxed $\mathbf{S}_{\mathbf{0}}$ geometry is formed by a broad band in the near IR, the highest excitation being at $2258 \mathrm{~nm}\left(\mathrm{f} \cdot 10^{-3}=140\right)$, and a shoulder centered at $780 \mathrm{~nm}$, with a smaller oscillator strength $\left(f \cdot 10^{-3}=33\right)$. The analysis of the composition of the computed excitations shows that the initial state involved in the electronic transitions is always the SOMO, while the final state is among one of the unoccupied MOs in the CB. As mentioned in the main text, the SOMO lies just below the LUMO in the unrelaxed $\mathbf{S}_{\mathbf{0}}$ geometry, so that very low excitation energies are able to populate the unoccupied MOs in the $\mathrm{CB}$, giving rise to a high intensity band in the near IR.

\section{S2.2 Absorption spectra of photoexcited holes}

Table S2 and Figure S5 show energies and oscillator strengths of selected excitations of a trapped hole, together with the composition in terms of the most relevant transitions and the involved MOs computed at the TD-B3LYP/3-21G* level of theory.

The simulated absorption spectrum of a trapped $\mathrm{h}^{+}$in the relaxed $\mathbf{D}_{1, \mathbf{h}}$ geometry shows only the first excitation in the IR at $1901 \mathrm{~nm}$, with a very low intensity $\left(\mathrm{f} \cdot 10^{-3}=0.3\right)$, while all remaining excitations are in the visible $(658-315 \mathrm{~nm})$. Overall, the absorption spectrum shows a broad absorption band, with two more intense peaks at 556 and $436 \mathrm{~nm}\left(\mathrm{f} \cdot 10^{-3}=6.6\right.$ and 5.5 , respectively) and a less intense one at $326 \mathrm{~nm}\left(\mathrm{f} \cdot 10^{-3}=3.6\right)$. Analysis of the composition of the computed excitations shows that the final state is always the SUMO (722 $\beta)$, while the initial state is one of the occupied MOs below the HOMO, formally constituting the VB. The only exception is the peak at $315 \mathrm{~nm}$ which is mainly constituted by electronic transitions to MOs lying at the bottom of the CB, rather than the SUMO in the band gap (see Table S2). Since the HOMO-LUMO gap is larger than the HOMO-SUMO gap by ca. $2.0 \mathrm{eV}$, low energy excitations inject the electron in the 
SUMO, leaving a hole in the VB. Conversely, the excitations in the near UV have the energy sufficient to inject the electron in the $\mathrm{CB}$, while the hole is at the top of the VB.

The excitation in the IR at $1901 \mathrm{~nm}$ is mainly composed by electronic transitions from occupied MOs lying very close in energy to the HOMO to the SUMO. In the visible region, the peak centered at $556 \mathrm{~nm}$ arises mainly from excitations 2, 5, 6 and 8, which have the highest oscillator strengths in the computed spectrum ( $\cdot 10^{-3}=7.5,2.8,6.9$ and even 12.8 , respectively). While excitations 2 and 5 are mainly composed by electronic transitions from the highest MOs (HOMO and HOMO-1) to the SUMO, excitations 6 and 8 have as initial states MOs lying deeper in energy, up to $0.5 \mathrm{eV}$ below the HOMO (i.e. HOMO-9, HOMO-10, HOMO-11, HOMO-14). The remaining part of the spectrum in the visible region is characterized by a greater number of excitations (ca. 80) very close in energy, but with lower oscillator strengths ( $\mathrm{f} \cdot 10^{-3}<4.3$, except for excitation 100 with $\mathrm{f} \cdot 10^{-3}=6.6$ ), thus suggesting a smaller density of states. The excitations with higher oscillator strength $\left(\mathrm{f} \cdot 10^{-3}=3.0-4.3\right)$ are $34,37,44,45$ and 51 and are mainly composed by electronic transitions from MOs lying ca. $0.8-1.2 \mathrm{eV}$ below the HOMO and showing a largely delocalized electron density.

As shown in Figure S3, the computed absorption spectrum of hole in the unrelaxed $\mathbf{S}_{\mathbf{0}}$ geometry is very different from that in the relaxed $\mathbf{D}_{\mathbf{1 , h}}$ geometry. The former shows a broad band in the near IR, characterized by a maximum intensity at $2000 \mathrm{~nm}\left(\mathrm{f} \cdot 10^{-3}=120\right)$ and a shoulder slowly decreasing in intensity at higher wavelengths, while in the visible region a quite narrow band centered at $709 \mathrm{~nm}$ is present, with a much lower oscillator strength $\left(\mathrm{f} \cdot 10^{-3}=40\right)$. The analysis of the composition of the computed excitations shows that the initial state involved in the electronic transitions is among one of the occupied MO in the VB, while the final state is always the SUMO.

The pronounced red-shift of the unrelaxed vs relaxed $\mathrm{h}^{+}$absorption spectrum can be understood by comparing the $(\mathrm{TiO} 2)_{38}{ }^{+} \mathrm{MOs}$ energy scheme. Since in the $\mathrm{h}^{+}$ground state geometry the SUMO lies only $0.40 \mathrm{eV}$ above the HOMO, the excitations starting from the occupied MOs in the VB and arriving to the SUMO are expected at lower energy with respect to that in the relaxed 
geometry, where the SUMO lies $2.50 \mathrm{eV}$ above the HOMO. The band in the IR is constituted by few excitations in the 2500-7600 $\mathrm{nm}$ wavelength range $\left(f \cdot 10^{-3}=2-12\right)$, involving MOs lying within $0.5 \mathrm{eV}$ below the HOMO. The most intense excitation in the absorption spectrum of the unrelaxed $\mathrm{h}^{+}$polaron $\left(6377 \mathrm{~nm}, \mathrm{f} \cdot 10^{-3}=12.3\right)$ is mainly composed by an electronic transition from the HOMO-2 to the SUMO. In the 1000-2500 nm wavelength range we computed ca. 40 excitations involving as initial states MOs lying 0.5-1.4 eV below the HOMO. Finally, in the wavelength range up to $600 \mathrm{~nm}$, excitations involving MOS lying 1.4-2.0 eV below the HOMO are involved as initial states.

Table S2. Excitation energy $(\mathrm{E}, \mathrm{eV})$, wavelength $(\lambda, \mathrm{nm})$, oscillator strength $\left(\mathrm{f} \cdot 10^{-3}\right)$ and composition of some representative doublet excitations $\left(D_{n}\right)$ in terms of $\beta$ MOs $(H=H O M O$; $\mathrm{SU}=\mathrm{SUMO})$ for the $\left(\mathrm{TiO}_{2}\right)_{38}{ }^{+}$cluster in the relaxed $\mathbf{D}_{\mathbf{1 , h}}$ geometry computed at the B3LYP/3-21G* level of theory in water solvent.

$\left.\begin{array}{|l|l|l|l|l|}\hline \mathbf{D}_{\mathbf{n}} & \mathbf{E}(\mathbf{e V}) & \lambda(\mathbf{n m}) & \mathbf{f \cdot 1 0 ^ { - 3 }} & \multicolumn{1}{c|}{\text { composition }} \\ \hline 2 & 1.88 & 658 & 7.5 & 91 \%(\mathrm{H} \rightarrow \mathrm{SU})+28 \%(\mathrm{H}-1 \rightarrow \mathrm{SU}) \\ \hline 6 & 2.14 & 578 & 6.9 & 105 \%(\mathrm{H}-9 \rightarrow \mathrm{SU})+18 \%(\mathrm{H}-10 \rightarrow \mathrm{SU}) \\ \hline 8 & 2.22 & 559 & 12.8 & 73 \%(\mathrm{H}-14 \rightarrow \mathrm{SU})+27 \%(\mathrm{H}-11 \rightarrow \mathrm{SU})+18 \%(\mathrm{H}-10 \rightarrow \mathrm{SU})\end{array}\right)$



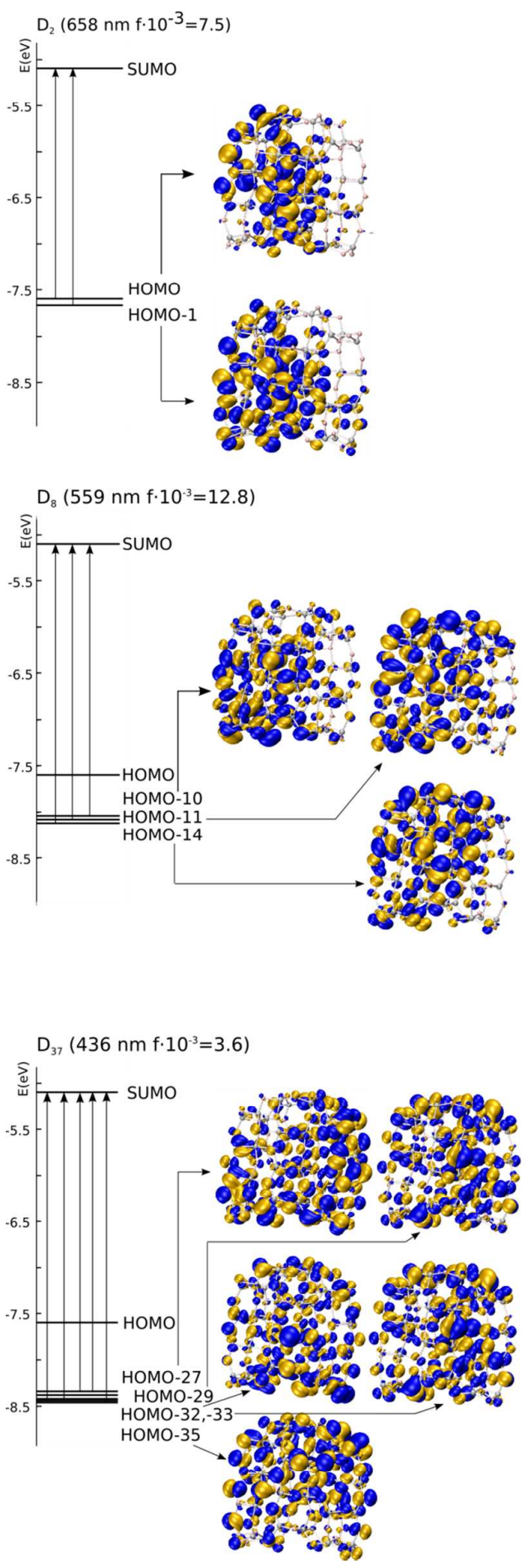

$D_{6}\left(578 \mathrm{~nm} \cdot 10^{-3}=6.9\right)$
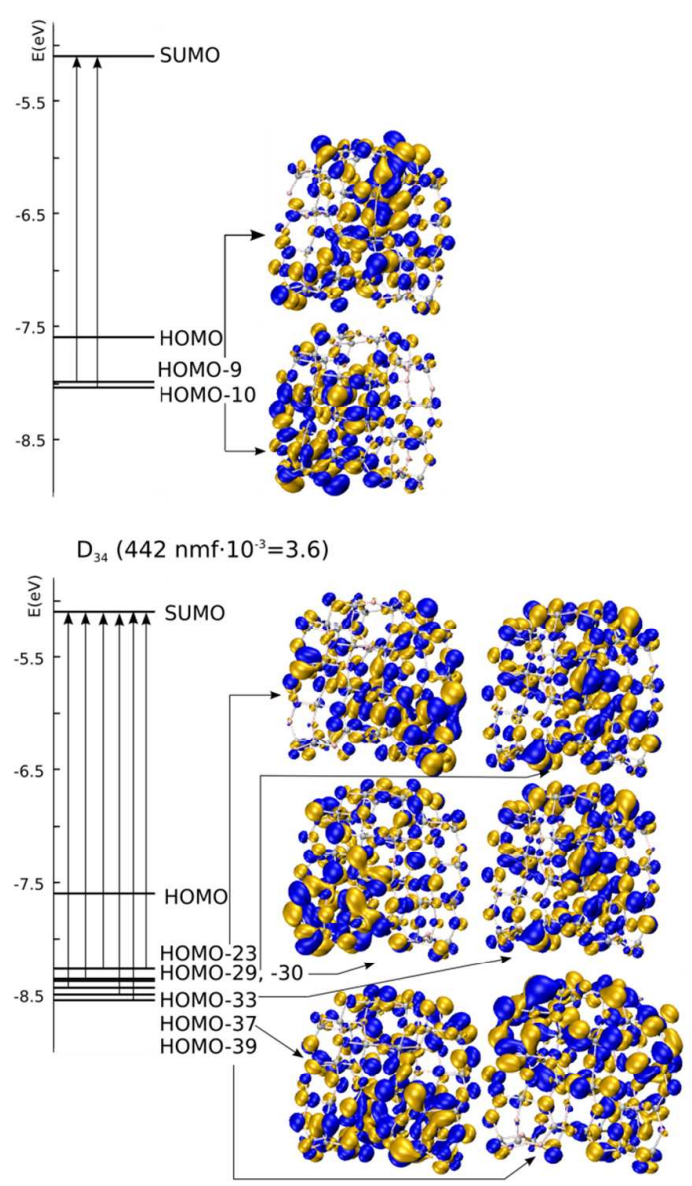

$D_{44}\left(413 \mathrm{~nm} f \cdot 10^{-3}=4.3\right)$

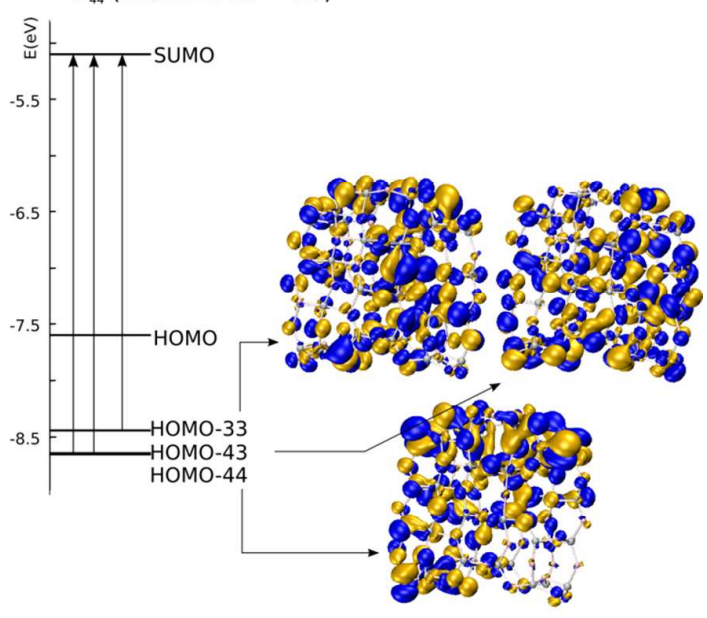

Figure S5. Representative excitations contributing to the spectrum of a trapped hole in the relaxed $\mathbf{D}_{\mathbf{1 , h}}$ geometry in terms of electronic transitions from occupied MOs in the VB to the SUMO computed at the TD-B3LYP/3-21G* level of theory. The electron wavefunctions of the involved occupied MOs are also shown (isovalue $=0.01 \mathrm{e} / \mathrm{a}$ 


\section{References}

(1) Vittadini, A.; Selloni, A.; Rotzinger, F. P.; Gratzel, M. Structure and Energetics of Water Adsorbed at $\mathrm{TiO}_{2}$ Anatase (101) and (001) Surfaces. 1998, 81, 2954-2957.

(2) Lundqvist, M. J.; Nilsing, M.; Persson, P.; Lunell, S. DFT Study of Bare and Dye-Sensitized $\mathrm{TiO}_{2}$ Clusters and Nanocrystals. Int. J. Quantum Chem. 2006, 106, 3214-3234.

(3) De Angelis, F.; Tilocca, A.; Selloni, A. Time-Dependent DFT Study of Fe(CN) ${ }_{6}^{4-}$ Sensitization of $\mathrm{TiO}_{2}$ Nanoparticles. J. Am. Chem. Soc. 2004, 126, 15024-15025.

(4) Persson, P.; Bergstrom, R.; Lunell, S. Quantum Chemical Study of Photoinjection Processes in DyeSensitized $\mathrm{TiO}_{2}$ Nanoparticles. J. Phys. Chem. B 2000, 104, 10348-10351.

(5) De Angelis, F.; Fantacci, S.; Selloni, A. Alignment of the Dye's Molecular Levels with the $\mathrm{TiO}_{2}$ Band Edges in Dye-Sensitized Solar Cells: a DFT-TDDFT Study. Nanotech. 2008, 19, 424002 (7pp).

(6) Cossi, M.; Rega, N.; Scalmani, G.; Barone, V. Structures, and Electronic Properties of Molecules in Solution with the C-PCM Solvation Model. 2003, 24, 669-681.

(7) Frisch, M. J.; Trucks, G. W.; Schlegel, H. B.; Scuseria, G. E.; Robb, M. A.; Cheeseman, J. R.; Scalmani, G.; Barone, V.; Mennucci, B.; Petersson, G. A., et al. Gaussian 09, Revision A.1; Gaussian, Inc.: Wallingford $\mathrm{CT}, 2009$.

(8) Tamaki, Y.; Furube, A.; Murai, M.; Hara, K.; Katoh, R.; Tachiya, M. Dynamics of Efficient ElectronHole Separation in $\mathrm{TiO}_{2}$ Nanoparticles Revealed by Femtosecond Transient Absorption Spectroscopy Under the Weak-Excitation Condition. Phys. Chem. Chem. Phys. 2007, 9, 1453-1460.

(9) Tamaki, Y.; Hara, K.; Katoh, R.; Tachiya, M.; Furube, A. Femtosecond Visible-to-IR Spectroscopy of $\mathrm{TiO}_{2}$ Nanocrystalline Films: Elucidation of the Electron Mobility before Deep Trapping. J. Phys. Chem. C 2009, 113, 11741-11746.

(10) Katoh, R.; Murai, M.; Furube, A. Transient Absorption Spectra of Nanocrystalline $\mathrm{TiO}_{2}$ Films at High Excitation Density. Chem. Phys. Lett. 2010, 500, 309-312. 